\title{
Prognostic value of systemic inflammation response index in patients with persistent human papilloma virus infection
}

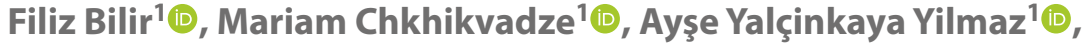 \\ Osman Kose2 ${ }^{2}$, Dagıstan Tolga Arıöz@ \\ ${ }^{1}$ Afyonkarahisar Health Science University Department of Gynecologic Oncology, Sakarya, Turkey \\ ${ }^{2}$ Department of Gynecologic Oncology, Sakarya Training and Research Hospital, Sakarya, Turkey
}

\begin{abstract}
Objectives: Persistent human papilloma virus (HPV) infection is a risk factor for the progression of cervical neoplasia into invasive carcinoma. Many inflammatory markers obtaining from hemogram parameters as platelets, monocytes, lymphocytes, and neutrophils or their ratios are still under investigation in recent decades, especially in the oncology era. Indeed, there have not been enough data about the relationship between these parameters and cervical cancer in the literature. Our primary aim was to investigate the possible relationship between the persistent HPV, which is one of the significant risk factors of cervical cancer, and these inflammatory markers. Further, we can add an easy follow-up parameter in women with persistent HPV infection.

Material and methods: The study included patients between 30-65 years old, tested positive for HPV, and afterward had an HPV control test between January 2015 and June 2020.

Results: The study included 114 HPV DNA-positive patients. The mean age was 43 (standard deviation 8.7), and 41 of them (36\%) had persistent HPV, but the remaining 73 (64\%) did not. The baseline neutrophil/lymphocyte ratio (NLR) value was 2.1, platelet/lymphocyte ratio (PLR) was 133 , monocyte/lymphocyte ratio (MLR) was 0.28 , and systemic inflammation response index (SIRI) was 0.9. All the parameters were significantly higher in the persistent HPV group compared to the non-persistent group. Patients who had 0.65 and under this had a significantly lower risk of persistent HPV.

Conclusions: Persistent HPV disease can be predicted with an elevated SIRI, NLR, and other hematologic parameters. So, we can closely follow up with these patients with different algorithms to prevent cervical cancer.

Key words: systemic inflammation response index; neutrophil to lymphocyte ratio; platelet to lymphocyte ratio, persistent HPV
\end{abstract}

Ginekologia Polska 2022; 93, 9: 705-709

\section{INTRODUCTION}

Human papilloma virus (HPV) is a DNA virus that affects the epidermis and mucosa, and it has been transmitted by skin-to-skin contact. There are more than 100 different types of HPV; the two major groups are high and low risks for cancer [1]. The prevalence of HPV infection was around $5 \%$ in Turkey, published by Gultekin et al., including more than 4 million women [2]. Other studies found different ranges according to the region and age between $13-45 \%[3,4]$.
Most HPV infections disappear within two years, but some persist, causing pre-malign cervical lesions as well as cervical cancer [5].

Persistent HPV infection is a risk factor for the progression of cervical neoplasia into invasive carcinoma [6]. Many studies showed that the persistence of HPV for a long time is crucial for cervical carcinogenesis. A systematic review found that HPV persistence is substantially linked to cervical intraepithelial neoplasia (CIN2-3) and, high-grade squamous

Corresponding author:

Filiz Bilir

Afyonkarahisar Health Science University Department of Gynecologic Oncology, 32 Evler mh 822 sk Serdivan, 54100 Sakarya, Turkey

e-mail:drflzyldz@hotmail.com

Received: 18.07.2021 Accepted: 11.09.2021 Early publication date: 1.02.2022

This article is available in open access under Creative Common Attribution-Non-Commercial-No Derivatives 4.0 International (CC BY-NC-ND 4.0) license, allowing to download articles and share them with others as long as they credit the authors and the publisher, but without permission to change them in any way or use them commercially. 
intraepithelial lesions (HSIL). Moreover, Longer durations of infection, longer test intervals for HPV, and higher-grade cervical illness all increased the magnitude of the effect of HPV persistence $[7,8]$.

Virchow found the connection between inflammation and cancer in 1863. Many hematologic parameters such as monocyte/lymphocyte ratio (MLR), neutrophil/lymphocyte ratio (NLR), platelet/lymphocyte ratio (PLR), systemic inflammation response index (SIRI) have been studied further [9$-12]$. Although some studies showed a relationship between cervical cancer and hematologic parameters, there was no study about persistent HPV relation and these parameters above, especially SIRI in the literature. Thus, this research aims to find a relation between persistent HPV with SIRI and other classic parameters.

\section{Objectives}

Persistent HPV infection is a risk factor for the progression of cervical neoplasia into invasive carcinoma. Many inflammatory markers obtaining from hemogram parameters as platelets, monocytes, lymphocytes, and neutrophils or their ratios are still under investigation in recent decades, especially in the oncology era. Indeed, there have not been enough data about the relationship between these parameters and cervical cancer in the literature. Our primary aim was to investigate the possible relationship between the persistent HPV, which is one of the significant risk factors of cervical cancer and these inflammatory markers. Further, we can add an easy follow-up parameter in women with persistent HPV infection.

\section{MATERIAL AND METHODS}

This study was designed in two gynecology centers in Turkey: Afyonkarahisar Health Sciences University and Sakarya Training and Research Hospitals. The research is a retrospective study approved by the ethics committee, including HPV positive female patients. The study included patients who were between 30-65 years old, tested positive for HPV, and afterward, had an HPV control test between January 2015 and June 2020. Patients who had an active infection had been vaccinated for HPV or had a history of malignancy were not included in this study.

All patients underwent HPV genotyping and liquid smear tests (Roche Cell-IVD) while having a gynecologic examination. The Bethesda System was used to report the cervical cytopathologic examination. Abnormal results in this system are squamous cell carcinoma, adenocarcinoma in situ (AIS), atypical squamous cells of undetermined significance (ASC-US), atypical squamous cells cannot exclude HSIL (ASC-H), low-grade squamous intraepithelial lesion (LSIL), high grade squamous intraepithelial lesion (HSIL), atypical glandular cells not otherwise specified
(AGC-NOS), atypical glandular cells, and suspicious for AIS or cancer (AGC-neoplastic). Our study used LGSIL and HGSIL because most of our pathology reports included these two scores, so we excluded the other rare score. Cervical intraepithelial neoplasia (CIN) classification shows the abnormal growth of cervical surface cells. We accepted CIN 1 lesion as LSIL and CIN 2/3 lesion as HSIL according to The College of American Pathology and the American Society of Colposcopy and Cervical Pathology reports to reduce the heterogeneity of centers and pathologists [13]. All patients controlled for HPV, in case the result was positive, the patient was followed up for at least 12 months and a retest was performed. In case of the same type of HPV being positive again, we accepted the patient as persistent for HPV.

\section{Measurement of hematologic parameters}

Complete blood counts and related parameters were obtained from the peripheral blood of patients before the diagnosis of HPV. An automated hematology analyzer counted peripheral neutrophils, lymphocytes, platelets, and monocytes. NLR and PLR were defined as absolute neutrophil/ /platelet count, respectively, divided by absolute lymphocyte count. MLR was defined as an absolute monocyte count divided by absolute lymphocyte count. SIRI was calculated as neutrophil count multiplied by monocyte count divided by lymphocyte count $(\mathrm{SIRI}=\mathrm{N} \times \mathrm{M} / \mathrm{L})$.

\section{Statistical analysis}

All analyses were done with SPSS version 22.0 (IBM Corporation, New York, USA). Women who had positive HPV results were being followed up, and in case the second test was positive, the patient was accepted as persistent for HPV. Otherwise, if the control test was negative, it was accepted as non-persistent HPV infection. Co-infection with another type of HPV, was not considered as persistent type. A normality test was performed for all data and determined with standard deviation or interquartile range as appropriate. Qualitative variables were analyzed with the chi-square test, and quantitative data analyzed with the Student $t$-test. Roc curve analysis calculated the cut off value for the SIRI score. $P$ values lower than 0.05 were considered statistically significant.

\section{RESULTS}

The study included 114 HPV DNA positive patients. The mean age was 43 [standard aviation (SD) 8.7], and 41 of them (36\%) had persistent HPV, but the remaining 73 (64\%) did not. Persistent HPV types were shown in Figure 1. The most common three types of persistent HPV were 16, 31, and 35 in our population. In this study, the mean hemoglobin level was $12.8 \mathrm{~g} / \mathrm{dL}$, white blood cell number was $7.7 \mathrm{~mm}^{3} \times 1,000$, 


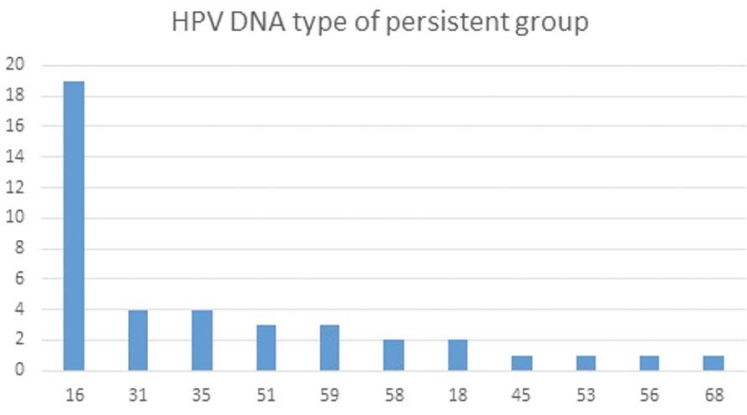

Figure 1. Persistent Human papilloma virus (HPV) DNA types

the neutrophil count was $4.6 \mathrm{~mm}^{3} \times 1,000$, and platelet count was $295 \mathrm{~mm}^{3} \times 1,000$.

The baseline NLR value was 2.1, PLR was 133, MLR was 0.28 , and SIRI was 0.9 . When we compare persistent and non-persistent groups, all four variables were significantly different and shown in Table 1. All the parameters were significantly higher in the persistent HPV group. The persistent HPV group had a significantly higher rate of HSIL/LSIL ratio (27/30 vs. $14 / 44)$ and the chi-square statistic is 6.7624 . The $p$ value is 0.00931 .

Roc-curve analysis for SIRI with 0.65 value has $95 \%$ sensitivity and $79 \%$ specificity with AUC 0.71 , shown in Figure 2. Patients who had 0.65 and under this SIRI value had a significantly lower risk of persistent HPV disease. One of 16 patients had a positive HPV in patients who had SIRI scores lower than 0.6 but, 40 of 98 patients had persistent HPV disease in a patient with a higher SIRI score (The chi-square statistic is 7.1359. The $p$ value is 0.0075 ).

\section{DISCUSSION}

This study found significantly higher NLR, PLR, and MLR values in patients with persistent HPV than the non-persistent HPV group. Although this is the first time in the literature, according to our research, SIRI scores are significantly higher in the persistent HPV group. This score could predict the persistence risk of HPV; therefore, it can be a valuable hematologic marker to follow up HPV-positive patients.

One of the most common causes of sexually transmitted infection is HPV in women and men. According to DNA sequence data, there are more than a hundred types of HPV, and they can be divided into high and low-risk types based on their association with pre-malign lesions and cervical cancer [14, 15]. The connection between HPV and cervical cancer has become well defined, and its contribution is more prominent than lung cancer with smoking [16]. It is accepted worldwide that more than $99 \%$ of cervical squamous cell cancer cases are related to HPV [17].

The systemic inflammation response index is a new biomarker calculated with peripheral blood neutrophil, monocyte, and lymphocyte counts. It can be used for the prognosis of patients with cancer, for instance, pancreatic [12], esophageal [10], and nasopharyngeal [18]. In a recent study, Chao et al. [9] found that SIRI was correlated with cervical cancer prognosis. They found a 1.25 cut-off value for SIRI. Above that, values were had worse outcomes, such as overall survival and recurrence. Higher NLR, PLR, and MLR values were significantly correlated with decreased overall survival for cer-

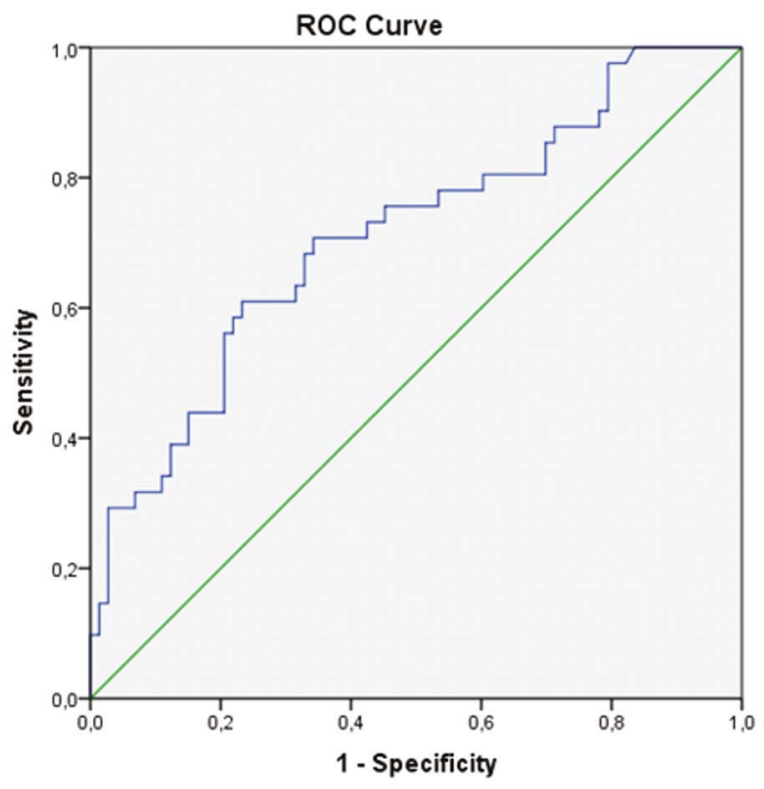

Diagonal segments are produced by ties

Figure 2. Receiver operating characteristic (ROC) curve anaylsis for systemic inflammation response index score

\begin{tabular}{|l|c|c|c|}
\hline \multicolumn{4}{|l|}{ Table 1. Comparison of hematologic parameters in persistent and non-persistent human papilloma virus (HPV) groups } \\
\hline Variable & Persistent HPV & Non-persistent HPV & p value \\
\hline Number & 41 & 73 & 0.0001 \\
\hline NLR & $2.6(1.2)$ & $1.8(0.5)$ & 0.005 \\
\hline PLR & $149(54)$ & $125(34)$ & 0.0001 \\
\hline MLR & $0.28(0.1)$ & $0.2(0.05)$ & 0.0001 \\
\hline SIRI & $1.2(0.5-5.7)$ & $0.85(0.3-2.6)$ & \\
\hline
\end{tabular}

NLR — neutrophil/lymphocyte ratio; PLR — platelet/lymphocye ratio; MLR — monocyte/lymphocye raito; SIRI — systemic inflammation response index 
vical cancer. The cut-off value was 2.8, 135, and 0.29, respectively [9]. Another large cohort study found higher NLR, PLR, and MLR values significantly related with worse overall survival in cervical cancer, cut-off values were $2.4,118,0.26$, respectively [19]. Our research also supports these results, and besides, as a new marker, SIRI could be predictive for persistent HPV infection. Our study's cut-off values were 2.6, 149, and 0.28, respectively, and 0.6 for SIRI. Nearly $10 \%$ of HPV infections tend to persist, most of them could clear spontaneously, and about $2 \%$ of them persist as a chronic disease and proceed to heterogenous dysplastic changes as LSIL or HSIL. The etiologic role of persistent infection with high risk-HPV in pre-malign lesions and cervical cancer is well known [20, 21].That's why determining high-risk HPV types and follow-up for persistence are essential to diagnose cancer and early treatment if possible. This study found that SIRI is an easy and cheap method to predict the disease's persistence.

Increased neutrophil counts following chemotherapy can cause a worse prognosis in biliary tract cancer [22]. Furthermore, peripheral absolute lymphocyte count has a predictive value for tumor shrinkage and survival in cervical cancer patients following the definitive chemoradiation. Besides, post-treatment lymphopenia was correlated with decreased overall survival $[23,24]$. In a meta-analysis including 26 studies and more than 10.000 patients diagnosed with gynecologic cancers, reported that NLR higher than 2.95 was correlated with worse overall survival (OS) [hazard ratio (HR) 1.65, 95\% confidence interval $(\mathrm{Cl}), \mathrm{p}<0.001$ ] [25]. To our knowledge, there is no study found any relation between the HPV and hematologic parameters other than oropharyngeal cancer. Our research found that persistent HPV can cause higher NLR, PLR, $M L R$, and SIRI values than the non-persistent type. We can all agree that it is easy to check these parameters while screening for HPV in the general population. According to the results, recommendations can be given to the patients such as short-term follow-up periods, different vaccination schedules, or detailed histologic analyses because of the higher value of those hematologic parameters more risk for cervical cancer.

It was not our primary aim, but there are much recent data about molecular and inflammatory pathways. HPV viruses aid carcinogenesis by exacerbating the accumulation of UV-induced DNA breaks and somatic mutations with the expression of viral oncoproteins E6 and E7. E6 oncoprotein make a complex with $\mathrm{p} 53$ tumor suppressor protein, E7 protein binds to retinoblastoma $(\mathrm{Rb})$ protein and both deploy the cell cycle checkpoints and cause a genomic instability then increase transformation rate of cells [26]. Furthermore, CD8+ T cells were limited to the stroma of dysplastic epithelium. They were missing in the lesioned epithelium of persistent cervical lesions and HPV-related malignancies; the presence of a cytotoxic CD8+T cell infiltration correlates with increased patient survival [27]. In addition, Patients with chronic HPV infection had greater local and circulating Treg frequencies than those with regressed lesions. E-cadherin, an adhesion molecule, plays a glue role in persistent HPV infection between the Langerhans cells and keratinocytes [28]. Lastly, more recent mechanism investigated keratinocytes (KCs) and antigen-presenting cells (APC). KCs are a component of the innate immune system and express several toll-like receptors (TLRs) which trigger type I interferon (IFN) release and proinflammatory cytokines such as tumor necrosis factor a (TNF- $a$ ) and interleukin $1 \beta$ (IL1- $\beta$ ) [29]. Active HPV infection and/or expression of E6/E7 oncoproteins were linked to depletion of intraepithelial LCs in cervical precancerous lesions and invasive malignancies.

Furthermore, collecting LCs in biopsy specimens is significantly associated with clearance of HPV infection in the cervix [30]. Also, it has shown that APCs were gradually disappeared through the epidermis in HPV-related skin warts [31]. Taken together, all these data can show that LCs has unique role to control the HPV-induced lesions, and inhibition of LC function and infiltration might be an immune escape mechanism of HPV infections [32].

We have some limitations, such as the retrospective study, and we did not compare it with the healthy control group. Still, our primary aim was to investigate differences between the persistent and non-persistent group of HPV. HPV colonization was only limited to the cervix. It does not consider anal or oral colonization. Also, a relatively small number of patients was another limitation of this study.

On the other hand, there is no standardization for the screening and follow-up of HPV infection. P16 detection is a helpful method for differentiating HSIL and LSIL in cervical lesion [33]. Besides, predicting persistent HPV infection to prevent cancer progression is not easy, and DNA based methods such as p16 detection are not usable worldwide. That's why our research is valuable, as the test is easily applicable in most clinics, and it could lead the way to plan the follow-up for the patients. However, prospective studies with larger patient populations are still needed.

\section{CONCLUSIONS}

In conclusion, persistent HPV disease can easily be predicted with elevated SIRI, NLR, and other hematologic parameters. Managing these patients should include different algorithms to prevent cervical cancer.

\section{Acknowledgements}

We do not have acknowledgments. 


\section{Conflict of interest}

None.

\section{REFERENCES}

1. Zhang $L, B i$, Deng $H$, et al. Human papillomavirus infections among women with cervical lesions and cervical cancer in Eastern China: genotype-specific prevalence and attribution. BMC Infect Dis. 2017; 17(1): 107, doi: 10.1186/s12879-017-2223-1, indexed in Pubmed: 28143439.

2. Gultekin M, Dundar S, Keskinkilic B, et al. How to triage HPV positive cases: results of four million females. Gynecol Oncol. 2020; 158(1): 105111, doi: 10.1016/j.ygyno.2020.04.698, indexed in Pubmed: 32362567.

3. Tunç SY, Onan MA, Turp AB, et al. Prevalence and types of cervical human papillomavirus among Turkish women and its relationship with demographic factors in a gynecology outpatient clinic. Eur J Gynaecol Oncol. 2016; 37(1): 53-58.

4. Beyazit F, SIlan F, Gencer M, et al. The prevelance of human papillomavirus (HPV) genotypes detected by PCR in women with normal and abnormal cervico-vaginal cytology. Ginekol Pol. 2018; 89(2): 62-67, doi: 10.5603/GP.a2018.0011, indexed in Pubmed: 29512809.

5. Demirtas D, Bilir C, Demirtas AO, et al. The effects of zoledronic acid on ECG: a prospective study on patients with bone metastatic cancer. Clin Cases Miner Bone Metab. 2017; 14(1): 35-39, doi: 10.11138/cc$\mathrm{mbm} / 2017.14 .1 .035$, indexed in Pubmed: 28740523.

6. Snijders PJF, Steenbergen RDM, Heideman DAM, et al. HPV-mediated cervical carcinogenesis: concepts and clinical implications. J Pathol. 2006; 208(2): 152-164, doi: 10.1002/path.1866, indexed in Pubmed: 16362994.

7. Koshiol J, Lindsay L, Pimenta JM, et al. Persistent human papillomavirus infection and cervical neoplasia: a systematic review and meta-analysis. Am J Epidemiol. 2008; 168(2): 123-137, doi: 10.1093/aje/kwn036, indexed in Pubmed: 18483125.

8. Trottier H, Mahmud SM, Lindsay L, et al. GSK HPV-001 Vaccine Study Group. Persistence of an incident human papillomavirus infection and timing of cervical lesions in previously unexposed young women. Cancer Epidemiol Biomarkers Prev. 2009; 18(3): 854-862, doi: 10.1158/10559965.EPI-08-1012, indexed in Pubmed: 19223559.

9. Chao B, Ju X, Zhang L, et al. A novel prognostic marker Systemic Inflammation Response Index (SIRI) for operable cervical cancer patients. Front Oncol. 2020; 10: 766, doi: 10.3389/fonc.2020.00766, indexed in Pubmed: 32477958.

10. Geng $Y$, Shao $Y$, Zhu D, et al. Systemic immune-inflammation index predicts prognosis of patients with esophageal squamous cell carcinoma: a propensity score-matched analysis. Sci Rep. 2016; 6: 39482, doi: 10.1038/srep39482, indexed in Pubmed: 28000729.

11. Tokunaga R, Sakamoto $Y$, Nakagawa $S$, et al. Comparison of systemic inflammatory and nutritional scores in colorectal cancer patients who underwent potentially curative resection. Int J Clin Oncol. 2017; 22(4): 740-748, doi: 10.1007/s10147-017-1102-5, indexed in Pubmed: 28213742.

12. Qi Qi, Zhuang $L$, Shen $Y$, et al. A novel systemic inflammation response index (SIRI) for predicting the survival of patients with pancreatic cancer after chemotherapy. Cancer. 2016; 122(14): 2158-2167, doi: 10.1002/cncr.30057, indexed in Pubmed: 27152949.

13. Darragh TM, Colgan TJ, Thomas Cox J, et al. Members of the LAST Project Work Groups. The lower anogenital squamous terminology standardization project for HPV-associated lesions: background and consensus recommendations from the College of American Pathologists and the American Society for Colposcopy and Cervical Pathology. Int J Gynecol Pathol. 2013; 32(1): 76-115, doi: 10.1097/PGP.0b013e31826916c7, indexed in Pubmed: 23202792

14. Burd EM. Human papillomavirus and cervical cancer. Clin Microbiol Rev. 2003; 16(1): 1-17, doi: 10.1128/CMR.16.1.1-17.2003, indexed in Pubmed: 12525422

15. Viruses in human cancers. Proc Assoc Am Physicians. 1999; 111(6): 581-587, doi: 10.1046/j.1525-1381.1999.99723.x, indexed in Pubmed: 10591087.

16. Franco EL. Cancer causes revisited: human papillomavirus and cervical neoplasia. J Natl Cancer Inst. 1995; 87(11): 779-780, doi: 10.1093/jnci/87.11.779, indexed in Pubmed: 7791221.
17. Walboomers JM, Jacobs MV, Manos MM, et al. Human papillomavirus is a necessary cause of invasive cervical cancer worldwide. J Pathol. 1999; 189(1): 12-19, doi: 10.1002/(SICI)1096-9896(199909)189:1<12::A ID-PATH431>3.0.CO;2-F, indexed in Pubmed: 10451482.

18. Chen $Y$, Jiang $W, X i D$, et al. Development and validation of nomogram based on SIRI for predicting the clinical outcome in patients with nasopharyngeal carcinomas. J Investig Med. 2019; 67(3): 691-698, doi: 10.1136/jim-2018-000801, indexed in Pubmed: 30127099.

19. Huang $\mathrm{H}$, Liu $\mathrm{Q}$, Zhu L, et al. Prognostic value of preoperative systemic immune-inflammation index in patients with cervical cancer. Sci Rep. 2019; 9(1): 3284, doi: 10.1038/s41598-019-39150-0, indexed in Pubmed: 30824727.

20. O'Brien PM, Bäckström T, Brown C, et al. Towards a consensus on diagnostic criteria, measurement and trial design of the premenstrual disorders: the ISPMD Montreal consensus. Arch Womens Ment Health. 2011; 14(1): 13-21, doi: 10.1007/s00737-010-0201-3, indexed in Pubmed: 21225438.

21. Nobbenhuis MA, Walboomers JM, Helmerhorst TJ, et al. Relation of human papillomavirus status to cervical lesions and consequences for cervical-cancer screening: a prospective study. Lancet. 1999; 354(9172): 20-25, doi: 10.1016/S0140-6736(98)12490-X, indexed in Pubmed: 10406360.

22. Cho KM, Park H, Oh DY, et al. Neutrophil-to-lymphocyte ratio, platelet-to-lymphocyte ratio, and their dynamic changes during chemotherapy is useful to predict a more accurate prognosis of advanced biliary tract cancer. Oncotarget. 2017; 8(2): 2329-2341, doi: 10.18632/oncotarget.13731, indexed in Pubmed: 27911876.

23. Hoskin PJ, Rojas AM, Peiris SN, et al. Pre-treatment haemoglobin and peripheral blood lymphocyte count as independent predictors of outcome in carcinoma of cervix. Clin Oncol (R Coll Radiol). 2014; 26(4): 179-184, doi: 10.1016/j.clon.2013.11.023, indexed in Pubmed: 24439272.

24. Wu ES, Oduyebo T, Cobb LP, et al. Lymphopenia and its association with survival in patients with locally advanced cervical cancer. Gynecol Oncol. 2016; 140(1): 76-82, doi: 10.1016/j.ygyno.2015.11.013, indexed in Pubmed: 26571200.

25. Ethier JL, Desautels DN, Templeton AJ, et al. Is the neutrophil-to-lymphocyte ratio prognostic of survival outcomes in gynecologic cancers? A systematic review and meta-analysis. Gynecol Oncol. 2017; 145(3): 584-594, doi: 10.1016/j.ygyno.2017.02.026, indexed in Pubmed: 28222899.

26. Jayshree RS. The immune microenvironment in human papilloma virus-induced cervical lesions-evidence for estrogen as an immunomodulator. Front Cell Infect Microbiol. 2021; 11: 649815, doi: 10.3389/fcimb.2021.649815, indexed in Pubmed: 33996630.

27. de Vos van Steenwijk PJ, Ramwadhdoebe TH, Goedemans R, et al. Tumor-infiltrating CD14-positive myeloid cells and CD8-positive T-cells prolong survival in patients with cervical carcinoma. Int J Cancer. 2013; 133(12): 2884-2894, doi: 10.1002/ijc.28309, indexed in Pubmed: 23740735.

28. Hubert $\mathrm{P}, \mathrm{Caberg} \mathrm{JH}$, Gilles $\mathrm{C}$, et al. E-cadherin-dependent adhesion of dendritic and Langerhans cells to keratinocytes is defective in cervical human papillomavirus-associated (pre)neoplastic lesions. J Pathol. 2005; 206(3): 346-355, doi: 10.1002/path.1771, indexed in Pubmed: 15852499.

29. Dai X, Tohyama M, Murakami $M$, et al. Epidermal keratinocytes sense dsRNA via the NLRP3 inflammasome, mediating interleukin (IL)-1 $\beta$ and IL-18 release. Exp Dermatol. 2017; 26(10): 904-911, doi: 10.1111/exd.13334, indexed in Pubmed: 28266737.

30. Shannon B, Yi TJ, Perusini S, et al. Association of HPV infection and clearance with cervicovaginal immunology and the vaginal microbiota. Mucosal Immunol. 2017; 10(5): 1310-1319, doi: 10.1038/mi.2016.129, indexed in Pubmed: 28120845.

31. Reva IV, Reva GV, Yamamoto T, et al. Distribution of antigen-presenting cells CD68 in papillomavirus infection in the skin. Bull Exp Biol Med. 2014; 157(1): 56-61, doi: 10.1007/s10517-014-2491-3, indexed in Pubmed: 24915947.

32. Bashaw AA, Leggatt GR, Chandra J, et al. Modulation of antigen presenting cell functions during chronic HPV infection. Papillomavirus Res. 2017; 4:58-65, doi: 10.1016/j.pvr.2017.08.002, indexed in Pubmed: 29179871.

33. Wentzensen N, Schwartz L, Zuna RE, et al. Performance of p16/Ki-67 immunostaining to detect cervical cancer precursors in a colposcopy referral population. Clin Cancer Res. 2012; 18(15): 4154-4162, doi: 10.1158/1078-0432.CCR-12-0270, indexed in Pubmed: 22675168. 